Sharif University of Technology
Scientia Iranica
Transactions E: Industrial Engineering
SCIENTIA

\title{
Robust optimization approach for pricing and shelf space decisions with uncertain demand
}

\author{
M.S. Sajadieh* and M. Danaei \\ Department of Industrial Engineering and Management Systems, Amirkabir University of Technology, Tehran, Iran.
}

Received 1 April 2019; received in revised form 18 March 2020; accepted 11 May 2020

\section{KEYWORDS}

Market potential;

Robust optimization;

Shelf space;

Uncertainty;

Pricing strategy.

\begin{abstract}
$\overline{\text { Abstract. Pricing and shelf space allocation are two main operational decisions in the }}$ retailing industry. This study simultaneously optimizes these two decisions in a supply chain with two manufacturers and one retailer under uncertainty of demand and price sensitivity parameters. Two manufacturers have different conditions in terms of parameters affecting demand and production. A robust optimization model and an exact solution approach are developed to find the optimal solution. The results show that price sensitivity, market potential, and production costs can have a synergistic effect on optimal values. Moreover, the market potential can rely on managerial decisions as it has a significantly positive impact on profitability. This parameter is found to be the most important tool for securing the profitability of supply chain members.
\end{abstract}

(C) 2022 Sharif University of Technology. All rights reserved.

\section{Introduction}

In retailing industry, shelves are considered as valuable assets where products compete on finite display space. Shelf Space Allocation (SSA) has a long history in literature and can be classified as illustrated in Table 1. As shown in Table 1, researches in literature may belong to more than one category. Although this study mainly concentrates on SSA and pricing scope, we briefly introduce some of the mentioned studies in Table 1 .

In the mathematical category, Corstjens and Doyle first involved the space and cross-space elasticity into a model and solved it using geometrical programming [2].

In the stochastic optimization category, Schaal

\footnotetext{
*. Corresponding author.

E-mail addresses: Sajadieh@aut.ac.ir (M.S. Sajadieh); Mohsendanaei91@aut.ac.ir (M. Danaei)
}

and Hübner by conducting numerical studies and applying a stochastic shelf-space optimization model showed that the impact of cross-space elasticities on shelf-space decisions and retail profit is very limited [29]. Fatemi Ghomi and Khalesi developed a mathematical model for shelf space optimization problems under stochastic demand [12]. Düsterhöft et al. determined optimal shelf quantities, an item optimal shelf segment, as well as optimal replenishment frequencies. They considered different shelf segments in different positions [13].

In the dynamic optimization category, Gilland and Heese studied a SSA model with two products under dynamic circumstances. A heuristic algorithm is used in which customers serve themselves from the store shelves [15].

In the simultaneous optimization category, Urban considered two different streams of research, i.e., SSA and inventory management. The model optimizes three decision variables: reorder point, order quantity, and display quantity on the shelf [19].

In SSA and shelf space design category, Ghazavi 
Table 1. Shelf space allocation literature classification.

\begin{tabular}{l} 
Mathematical optimization \\
\hline •Deterministic models: Anderson and \\
Amato [1], Corstjens and Dolye [2,3], Bultez \\
and Naert [4], Yang [5], Hwang et al. [6], \\
Hansen et al. [7], Castelli and Vanneschi [8], and \\
Zhou and Piramuthu [9]. \\
•Stochastic models: Amit et al. [10], \\
Hübner and Schaal [11], Fatemi Ghomi \\
and Khalesi [12], and Düsterhöft et al. [13]. \\
•Dynamic models: Corstjens and Doyle \\
[3], Gajjar and Adil [14], and Gilland and Heese [15]. \\
•SSA in supply chains chain: Martin-Herran \\
and Taboubi [16], and Hariga and Al-Ahmari [17]. \\
•Simultaneous optimization \\
•SSA and pricing \\
•SSA and trade promotion: \\
Tsao et al. [18]. \\
•SSA and inventory control: Urban [19], \\
Maiti and Maiti [20], Hariga et al. [21], \\
Ramaseshan et al. [22], Bai and \\
Kendall [23], Gao and Yu [24], and \\
Baron et al. [25]. \\
et al. [26], Ghazavi and Lotfi [27], and Flamand [28]. \\
\hline
\end{tabular}

and Lotfi presented a replacement function that integrates demand. This function simulates methods to anticipate customers movements within the store. The shelf level, the shelf space, and substantial-and-random utilities of stores zones are also taken into account, simultaneously [27]. Flamand et al. also developed a model that jointly examines assortment planning and store-wide SSA decisions [28].

\subsection{SSA and pricing}

Pricing optimization is an inseparable part of any system (for instance Shafiee-Gol et al. [30] and Sadjadi et al. [31]). On the other hand, SSA is a main operational issue that can affect retailer profit. The interactions of pricing and SSA in the retailing industry have then motivated a considerable number of researches. Although several models and approaches have been developed to deal with SSA and pricing problems, most of them focus on deterministic models. However, in real circumstances, the products demand uncertainty complicates these decisions. As mentioned before, in this study we focus on the literature that incorporates pricing decisions into SSA.

Reyes and Frazier developed an SSA model that takes two conflicting objectives into consideration, i.e., customer service factors and profitability [32]. Murray et al. then developed a model that simultaneously optimizes the preferences of the product display, the location of the shelves, and the price of products placed in a category, while considering the height and width of each shelf [33]. Tan and Wang investigated sharing revenue between contracts and its effect on pricing as well as shelf space decisions. They showed that a well-designed revenue-sharing contract will increase manufacturing profits [34].

Kurtulus and Toktay investigated a competitive model in which the two manufacturers offer different products to one retailer. The retailer determines the shelf space allocated to the retail category. They assumed symmetric demand potential, cost, and crossprice sensitivities for two manufacturers. Based on the final results, two products obtain the same shelf space, and their wholesale and retail prices are similar [35]. Leng et al. investigated space-exchange in retail problems. To access more customers, two retailers can exchange shelf space. They concluded that a game strategy can be implemented for both retailers, if and only if their stores are large enough to serve more than one-half of their customers [36].

$\mathrm{Li}$ et al. used game theory where demand is affected by competition among supply chain members. They used a demand function that involves product pricing as well as SSA [37]. Eisend studied several new empirical generalizations about shelf space elasticity. He found that the application of shelf space variation is a useful tool for managers and decision-makers in marketing [38].

Wang et al. proposed a pricing and SSA model derived from Kurtulus and Toktay model. In the developed model, the production cost and cross-price sensitivity of the two products are not the same. The non-symmetric market potential is assumed for both products [39]. Hübner and Schaal considered a model to maximize retailer profit by choosing the number of facings and their shelf position under the assumption of limited space when demand is stochastic. They assumed that the number and position of the product is affected by demand and solved the model with heuristic methods [40].

Schaal and Hübner optimized the assortment and allocated quantities for each product in a retail shelf. The model accounts for stochastic demand, substitution, and space-elasticity effects [29]. Moon et al. took into account joint product line decisions. They assumed dynamic substitutions are derived by the valuations that customers place on the products and the availability of each product type. In order to maximize retailer profit, an integer programming approach is developed [41]. Table 2 shows a summary of the relevant modeling and solution approaches for 


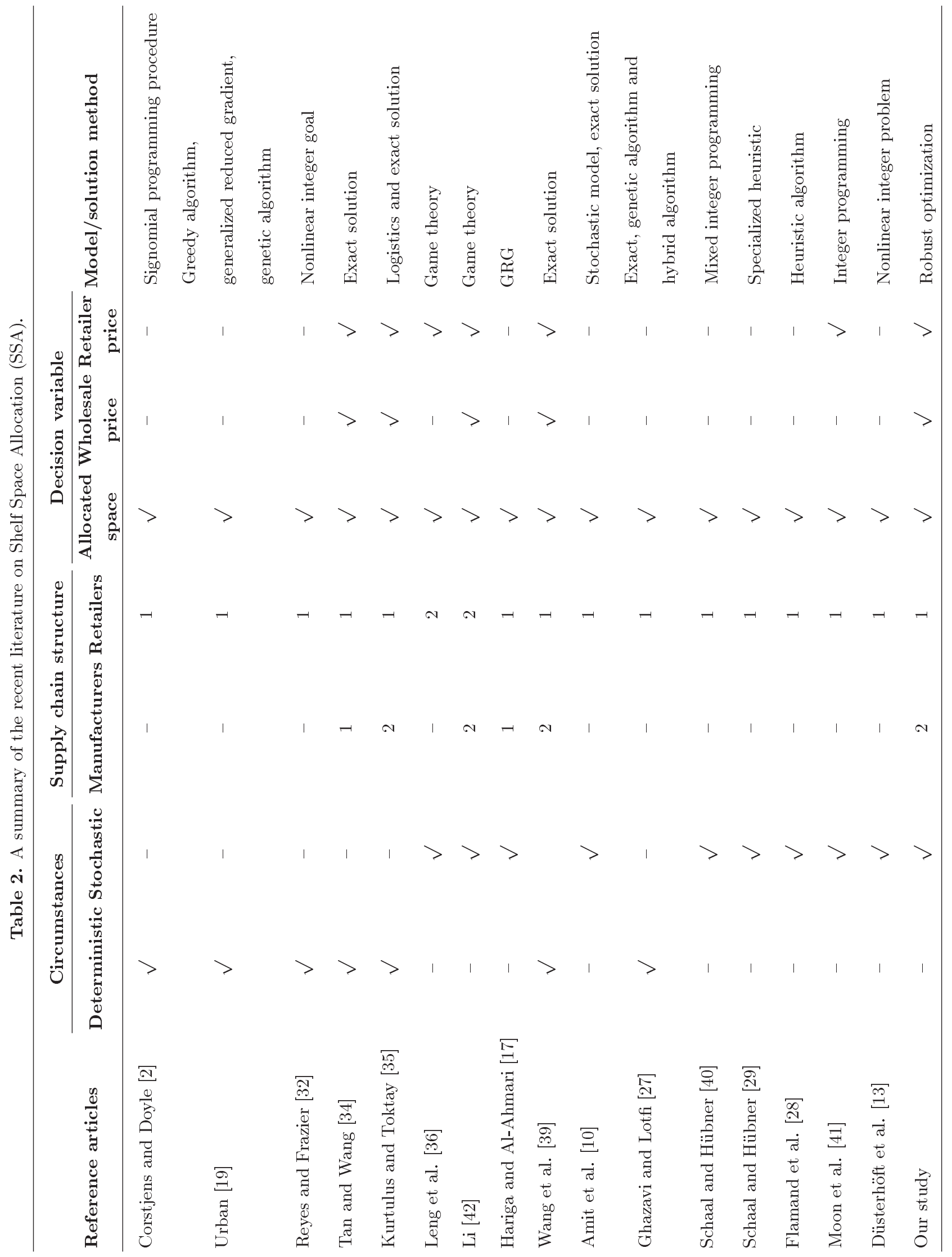


SSA. As shown in Table 2, there are few studies that look at how to decide on SSA and pricing policy simultaneously when there is uncertainty. However, in reality, critical parameters such as customer demand as well as price sensitivity could not be deterministic. Since pricing and shelf space decisions that are taken under uncertainty can affect all supply chain members, the robustness of these decisions is crucial.

This study employs a robust approach to consider uncertainties and maximize retailer profit. Using robust optimization to maximize retailer profits in a similar supply chain with a similar hypothesis has not been studied before. There is no prior study that uses robust optimization to maximize a retailer profit in a supply chain with two manufacturers and one retailer. The purpose of this research is to simultaneously optimize pricing and shelf space decisions under demand uncertainty. The simplicity of implementing the model allows the decision-makers to manage pricing and allocation decisions to achieve maximum profit.

The rest of the paper is structured as follows: In Section 2, the background of robust optimization formulation is presented. Section 3 presents the problem definition and formulation. Numerical analysis is presented in Section 4. Finally, conclusions are presented in Section 5.

\section{Robust optimization}

The robust optimization realizes uncertain parameters by defining different scenarios. The purpose of this method is to seek out a robust solution, that assures all specified scenarios are "close" to the optimum with regards to altering input data [43]. To cope with real-world problems containing noisy, erroneous data, methods such as robust optimization were developed in some cases. Mulvey et al. introduced the concept of stochastic robust optimization to control the trade-off between cost and its variability in stochastic program$\operatorname{ming}$ [44].

In the following, the framework of robust optimization is briefly represented [45]. Firstly, let $x \in R^{n 1}$ be a vector of the design variables and $y \in R^{n 2}$ be a vector of control variables. Then the form of the robust optimization model is as follows:

$$
\min c^{T} x+d^{T} y
$$

s.t.:

$$
\begin{aligned}
& A x=b, \\
& B x+C y=e, \\
& x, y \geq 0,
\end{aligned}
$$

where $B, C$, and $e$ represent the random technological constant matrix and right-hand side vector, respectively. Suppose a limited set of scenarios $\Omega=\{1,2, \ldots, \xi\}$ to consider uncertain parameters. With each scenario $\xi \in \Omega$ we associate the subset $\left\{d_{\xi}, B_{\xi}, C_{\xi}, e_{\xi}\right\}$ and the probability of the scenario $p_{\xi}\left(\sum \xi p_{\xi}=1\right)$.

Leung et al. introduced a solution for the proposed model that is feasible as well as optimal for all defined scenarios where $\xi \in \Omega$. The robust optimization model is formulated as follows [46]:

$$
\min \sigma\left(x, y_{1}, y_{2}, \ldots, y_{\xi}\right)+\omega\left(\delta_{1}, \delta_{2}, \ldots, \delta_{\xi}\right)
$$

s.t.:

$$
\begin{aligned}
& A x=b, \\
& B_{\xi} x+C_{\xi} y+\delta_{\xi}=e_{\xi}, \quad \forall \xi \in \Omega, \\
& x \geq 0, \quad y_{\xi} \geq 0, \quad \delta_{\xi} \geq 0, \quad \forall \xi \in \Omega .
\end{aligned}
$$

Due to parameter uncertainty, the model could also be infeasible for some scenarios. Therefore, $\delta_{\xi}$ presents the infeasibility of the model under scenario $\xi$. If the model is feasible, $\delta_{\xi}$ will be equal to 0 . Otherwise, $\delta_{\xi}$ will be assigned a positive value. In Eq. (5), the first term represents solution robustness, which takes into account the firm desire for low costs and the degree of its risk aversion, while the second term represents model robustness, which penalizes solutions that fail to meet demand in a scenario or violate other physical restrictions [43]. Mulvey et al. used the following relation [44]:

$$
\sigma(o)=\sum_{\xi \in \Omega} p_{\xi} \psi_{\xi}+\lambda \sum_{\xi \in \Omega} p_{\xi}\left(\psi_{\xi}-\sum_{\xi^{\prime} \in \Omega} p_{\xi^{\prime}} \psi_{\xi^{\prime}}\right)^{2} .
$$

It present solution robustness, where $\psi_{\xi}=f\left(x, y_{\xi}\right)$ is a cost or benefit function for scenario $\xi$. Parameter $\lambda$ denotes the weight placed on solution variance in which the solution is less sensitive to variations in the data under all scenarios as $\lambda$ increases. Hwang et al. indicated that to minimize Objective (9), the robust optimization model needs a great deal of computation. They proposed the following equation [26]:

$$
\sigma(o)=\sum_{\xi \in \Omega} p_{\xi} \psi_{\xi}+\lambda \sum_{\xi \in \Omega} p_{\xi}\left|\psi_{\xi}-\sum_{\xi^{\prime} \in \Omega} p_{\xi^{\prime}} \psi_{\xi^{\prime}}\right| .
$$

Although Objective (10) is a non-linear function, by converting the problem into a linear programming model with a linear objective function and linear constraints, Wagner claimed that it can be optimized by defining two non-negative deviational variables [47]. Instead of minimizing the sum of absolute deviations in Eq. (10), two deviational variables are minimized under original constraints and additional soft constraints that 
provide positive values of the difference inside the absolute functions, as supported by Leung et al. [46]. $\mathrm{Yu}$ and $\mathrm{Li}$ introduced an efficient method by developing Li study $[48,42]$. $\mathrm{Yu}$ and $\mathrm{Li}$ model framework is designed to minimize the objective function.

$$
\begin{aligned}
\min Z= & \sum_{\xi \in \Omega} p_{\xi} \psi_{\xi} \\
& +\lambda \sum_{\xi \in \Omega} p_{\xi}\left[\left(\psi_{\xi}-\sum_{\xi^{\prime} \in \Omega} p_{\xi^{\prime}} \psi_{\xi^{\prime}}\right)+2 \theta_{\xi}\right]
\end{aligned}
$$

s.t.:

$$
\begin{aligned}
& \psi_{\xi}-\sum_{\xi \in \Omega} p_{\xi} \psi_{\xi}+\theta_{\xi} \geq 0, \quad \forall \xi \in \Omega, \\
& \theta_{\xi} \geq 0, \quad \forall \xi \in \Omega .
\end{aligned}
$$

It can be verified that if $\psi_{\xi}-\sum_{\xi \in \Omega} p_{\xi} \psi_{\xi} \geq 0$, then $\theta_{\xi}=0$. On the other hand, if $\psi_{\xi}-\sum_{\xi \in \Omega} p_{\xi} \psi_{\xi} \leq 0$, then $\theta_{\xi}=\sum_{\xi \in \Omega} p_{\xi} \psi_{\xi}-\psi_{\xi}$. To penalize violations of the control constraints under a number of scenarios, the following model is used. Using the weight $\omega$, the trade-off between solution robustness measured from the primary term $\sigma(o)$ and model robustness measured from the penalty term $\rho(o)$ can be modeled under the Multiple-Criteria Decision-Making (MCDM) process.

$$
\begin{aligned}
\min Z= & \sum_{\xi \in \Omega} p_{\xi} \psi_{\xi}+\lambda \sum_{\xi \in \Omega} p_{\xi} \\
& {\left[\left(\psi_{\xi}-\sum_{\xi^{\prime} \in \Omega} p_{\xi^{\prime}} \psi_{\xi^{\prime}}\right)+2 \theta_{\xi}\right]+\omega \sum_{\xi \in \Omega} p_{\xi} \delta_{\xi} }
\end{aligned}
$$

\section{Problem definition}

A supply chain with two manufacturers and one retailer is considered. Manufacturers $A$ and $B$ produce and sell products $A$ and $B$ to a retailer at wholesale prices $w_{A}$ and $w_{B}$, respectively. The retailer then puts the products in shelf space $S$ and sells them at retail prices $p_{A}$ and $p_{B}$. Since products $A$ and $B$ belong to the same category (for example, breakfast cereals, soft drinks, and detergents), they can be replaced or substituted for one another. However, they have different market potentials and price sensitivity parameters. According to Shubik and Levitan [49], the demand function used is:

$$
\begin{aligned}
& q_{A}=a-p_{A}+\theta_{A}\left(p_{B}-p_{A}\right), \\
& q_{B}=b-p_{B}+\theta_{B}\left(p_{A}-p_{B}\right),
\end{aligned}
$$

where, $q_{A}$ and $q_{B}$ are the demand of products $A$ and $B$, respectively. Moreover, $a$ and $b$ represent the market potentials (the amount of demand regardless of its price). Parameters $\theta_{A}$ and $\theta_{B}$ are price sensitivity parameters, $\theta_{A}, \theta_{B} \in[0,1]$.

In the real-world, price sensitivity parameter can be under uncertainty due to technological, political, economic, and social factors. Obsolete technologies, for example, are not acceptable, even if they are inexpensive. In contrast, there are users willing to pay high value to possess products of the latest technology. Political factors like war and sanctions can also affect the price sensitivity of products.

In this study, we take into account the pricing and allocation decisions of shelf space where parameters $\theta_{A}$ and $\theta_{B}$ are uncertain under various scenarios. A set of scenarios $\Omega=\{1,2, \ldots, \xi\}$ are then defined with the probability of $\rho_{\xi}$ for scenario $\xi \in \Omega\left(\sum_{\xi \in \Omega} \rho_{\xi}=1\right)$.

The structure of the supply chain is shown in Figure 1. The retailer first outlines the available shelf space for two products and informs the manufacturers. Manufacturers then adjust their best wholesale prices in a competitive condition. Finally, the retailer determines retail prices according to wholesale prices.

In the next section, the problem is solved and optimal wholesale and retail prices, as well as the amount of allocated space, are obtained, using the backward induction method.

\subsection{Notation}

In this paper, the following parameters and decision variables are used:

\section{Parameters}

a Market potentials for product $A$

$b \quad$ Market potentials for product $B$

$\theta_{A \xi} \quad$ Cross-price sensitivity parameters for product $A$ under scenario $\xi$

$\theta_{B \xi} \quad$ Cross-price sensitivity parameters for product $B$ under scenario $\xi$

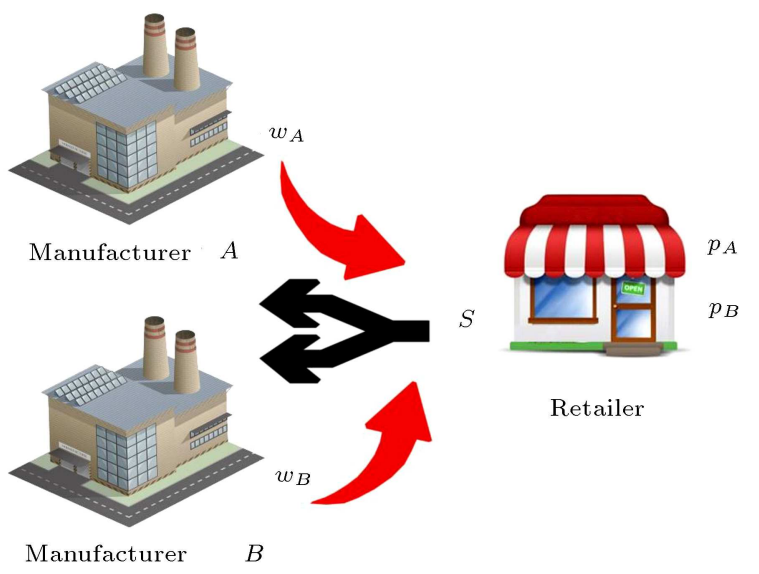

Figure 1. Considered supply chain in the study. 
$c_{A} \quad$ Unit production costs of product $A$

$c_{B} \quad$ Unit production costs of product $B$

$k \quad$ Shelf space opportunity cost

$\rho_{\xi} \quad$ Occurrence probability of scenario $\xi$

\section{Decision variables}

$p_{A} \quad$ Retail price for product $A$

$p_{B} \quad$ Retail price for product $B$

$w_{A} \quad$ Wholesale price for product $A$

$w_{B} \quad$ Wholesale price for product $B$

$S \quad$ Shelf space, i.e., the number of units of the product that can be stored on the shelf

$q_{A} \quad$ Demand for product $A$

$q_{B} \quad$ Demand for product $B$

$Z_{\xi} \quad$ Retailer profit under scenario $x i(1,2, \ldots, \xi)$

\subsection{Model development}

In this section, a robust model is developed to solve the pricing and shelf space problem for a non-symmetric supply chain.

\subsubsection{Retail price decisions}

Assuming that the shelf space $S$ and wholesale prices $w_{A}$ and $w_{B}$ are known, the retailer decides about retail prices $p_{A}$ and $p_{B}$ to maximize his profit. The problem model at this stage is as follows:

$$
\max \prod_{n}=\left(p_{A}-w_{A}\right) q_{A}+\left(p_{B}-w_{B}\right) q_{B}
$$

s.t.:

$$
q_{A}+q_{B} \leq S
$$

By respectively substituting Eqs. (15) and (16) into Eqs. (17) and (18), the robust optimization model is presented as follows:

$$
\begin{aligned}
\max \prod_{n} & =\sum_{\xi \in \Omega} p_{\xi} Z_{\xi}-\lambda \sum_{\xi \in \Omega} p_{\xi}\left[\left(\psi_{\xi}\right.\right. \\
& \left.\left.-\sum_{\xi^{\prime} \in \Omega} p_{\xi^{\prime}} \psi_{\xi^{\prime}}\right)+2 u_{\xi}\right]-\psi \sum_{\xi \in \Omega} p_{\xi} \varepsilon_{\xi}
\end{aligned}
$$

s.t.:

$$
\begin{aligned}
Z_{\xi}= & a p_{A}-p_{A}^{2}+p_{A} \theta_{A \xi} p_{B}-p_{A}^{2} \theta_{A \xi} \\
& -w_{A}\left(a-p_{A}+\theta_{A \xi} p_{B}-\theta_{A \xi} p_{A}\right) \\
& +b p_{B}-p_{B}^{2}+p_{B} \theta_{B \xi} p_{A}-p_{B}^{2} \theta_{B \xi} \\
& -w_{B}\left(b-p_{B}+\theta_{B \xi} p_{A}-\theta_{B \xi} p_{B}\right), \quad \forall \xi \in \Omega,
\end{aligned}
$$

$$
\begin{gathered}
a-p_{A}+\theta_{A \xi} p_{B}-\theta_{A \xi} p_{A}+b-p_{B}+\theta_{B \xi} p_{A} \\
-\theta_{B \xi} p_{B}+\varepsilon_{\xi} \leq S, \quad \forall \xi \in \Omega, \\
Z_{\xi}-\sum_{\xi^{\prime} \in \Omega}\left(p_{\xi^{\prime}} \psi_{\xi^{\prime}}\right)+u_{\xi} \geq 0, \quad \forall \xi \in \Omega, \\
p_{A}, p_{B} \geq 0, \quad u_{\xi}, \varepsilon_{\xi} \geq 0, \quad \forall \xi \in \Omega .
\end{gathered}
$$

The first and second terms in Eq. (19) are the mean value and variance of the objective function, respectively. The last term in Eq. (19) measures the model robustness with reference to infeasibility associated with Constraints (21) under scenario $\xi$. Moreover, Eq. (20) are defined just for formulation convenience. Constraints (21) are used to control the shelf space limitation. Eq. (22) are the auxiliary constraints for linearization defined in Eq. (14). Constraints (23) specify non-negative variables.

The above robust model is complicated when incorporating the effects of non-symmetric market potentials, production costs, and cross-price sensitivity parameters. For this reason, the optimal results are fitted by considering the effect of various parameters on the solution. To ensure that optimized solutions are global, the proof of objective function concavity is presented in Appendix A. The proposed model is solved by GAMS software for different values of parameters.

The optimistic, pessimistic, and normal scenarios are considered with different price sensitivity parameters. In an optimistic scenario, the price sensitivity of both products is low, ranging from 0 to 0.2 . In the pessimistic scenario, where customers are very sensitive to price, the parameter is considered between 0.8 to 1 . The price sensitivity parameter for the normal scenario is 0.2 to 0.8 .

Then, all possible combinations related to the probability of occurrence of scenarios are taken into account. Considering all possible combinations, optimal solutions are obtained for 1240 runs. We then fit optimal retail prices using SPSS software through a wide range of optimal solutions for three scenarios. Rsquared as the coefficient of determination is 0.98 and 0.898 for $p_{A}$ and $p_{B}$, respectively. We can conclude that the differences between the observed values and the model predicted values are small since the higher the R-squared, the better the model fits our data. We obtained optimal retail prices for special scenarios including random data based on SPSS results.

Given $S, w_{A}$, and $w_{B}$, the optimal retail prices $p_{A}^{*}$ and $p_{B}^{*}$ are then obtained as follows:

$$
\begin{aligned}
p_{A}^{*}= & 1.139 a+0.919 b+0.081 w_{A} \\
& -0.077 w_{B}-0.448 S-0.101 \sum_{\xi} \rho_{\xi} \theta_{A \xi},
\end{aligned}
$$




$$
\begin{aligned}
p_{B}^{*}= & 1.028 b+0.681 a-0.059 w_{A} \\
& +0.057 w_{B}-0.395 S-0.04 \sum_{\xi} \rho_{\xi} \theta_{A \xi} \\
& -0.095 \sum_{\xi} \rho_{\xi} \theta_{B \xi} .
\end{aligned}
$$

\subsubsection{Wholesale price decisions}

At this stage, each manufacturer decides on the wholesale price to maximize his profit assuming that the amount of shelf space $S$ is available. Profit functions for manufactures are as follows:

$$
\begin{aligned}
& \prod_{A}=\left(w_{A}-c_{A}\right) q_{A}, \\
& \prod_{B}=\left(w_{B}-c_{B}\right) q_{B} .
\end{aligned}
$$

By substituting Eqs. (24) and (25) into Eqs. (15) and (16), respectively, decision variable $q_{A}^{*}$ and $q_{B}^{*}$ are obtained. Then, by substituting $q_{A}^{*}$ and $q_{B}^{*}$ into Eqs. (26) and (27), and considering $\partial \prod_{A} / \partial w_{A}=0$ and $\partial \prod_{B} / \partial w_{B}=0$, the optimal wholesale price for each manufacturer are calculated.

Given $S$, optimal wholesale price $w_{A}^{*}$ and $w_{B}^{*}$ can be determined as follows:

$$
\begin{aligned}
w_{A}^{*}= & \frac{c_{A} E_{1}+c_{B} E_{2}+E_{3}+a E_{4}+b E_{5}+S E_{6}}{E_{9} E_{8}} \\
& +\frac{E_{10}}{E_{9}}, \\
w_{B}^{*}= & \frac{c_{A} F_{2}+c_{B} F_{3}+F_{4}+a F_{5}+b F_{6}+S F_{7}}{F_{1}} .
\end{aligned}
$$

Expressions $E_{1}$ to $E_{10}$ and $F_{1}$ to $F_{7}$ can be found in Appendix B.

\subsection{Retail shelf space size decision}

The retailer decides on the amount of shelf space available to supply different products. Taking into account the opportunity cost of shelf space the profit function for the retailer is as follows:

$$
\prod_{r}=\prod_{n}-k S^{2}
$$

where $k$ is a positive constant indicating the shelf space opportunity cost parameter. To calculate the optimal shelf space, we substitute Eqs. (25) and (26) into Eq. (30), and by taking the partial derivative of the equation with respect to $S$, the optimal space for the shelf is calculated as follows:

$$
S^{*}=\frac{0.5\left[c_{A} G_{1}+c_{B} G_{2}+a G_{3}+b G_{4}+G_{5}\right]}{k G_{6}+G_{7}} .
$$

Expressions $G_{1}$ to $G_{7}$ can be found in Appendix B.

\section{Numerical analysis}

In this section, to show the effect of various parameters on the optimal result, three scenarios are considered. In numerical analysis, we surveyed real data used in similar studies (e.g., $[39,46])$ and generated our random numbers in the range of real data used in the literature.

We first discuss the effect of market potential on optimal solutions. Tables $3-5$ provide an insight into the output data characteristics by setting $\lambda=0.01$ and model robustness $\psi=2000$.

Assuming $k=0.5, b=10, c_{A}=c_{B}=1, \sum_{\xi \in \Omega} \rho_{\xi}$

\begin{tabular}{|c|c|c|c|c|c|c|c|c|c|c|}
\hline \multirow[b]{2}{*}{$a / b$} & \multicolumn{4}{|c|}{ Retailer } & \multicolumn{3}{|c|}{ Manufacturer $A$} & \multicolumn{3}{|c|}{ Manufacturer $B$} \\
\hline & $S^{*}$ & $p_{A}^{*}$ & $p_{B}^{*}$ & $\prod_{r}$ & $\boldsymbol{w}_{A}^{*}$ & $q_{A}^{*}$ & $\prod_{A}$ & $\boldsymbol{w}_{B}^{*}$ & $q_{B}^{*}$ & $\prod_{B}$ \\
\hline 0.2 & 6.31 & 3.49 & 5.51 & 5.11 & 2.32 & 0.09 & 0.12 & 3.69 & 2.17 & 5.83 \\
\hline 0.4 & 8.06 & 4.23 & 5.71 & 8.68 & 2.46 & 1.31 & 1.91 & 3.41 & 2.11 & 5.09 \\
\hline 0.6 & 9.80 & 4.99 & 5.95 & 13.00 & 2.64 & 1.62 & 2.67 & 3.24 & 2.07 & 4.64 \\
\hline 0.8 & 11.55 & 5.72 & 6.22 & 18.37 & 2.78 & 1.84 & 3.28 & 3.11 & 2.01 & 4.24 \\
\hline 1.0 & 13.29 & 6.49 & 6.49 & 24.71 & 2.97 & 1.97 & 3.89 & 2.97 & 1.97 & 3.89 \\
\hline 1.2 & 15.04 & 7.21 & 6.72 & 32.61 & 3.08 & 2.12 & 4.41 & 2.78 & 1.93 & 3.44 \\
\hline 1.4 & 16.77 & 7.93 & 6.96 & 41.70 & 3.19 & 2.23 & 4.88 & 2.59 & 1.88 & 2.99 \\
\hline 1.6 & 18.52 & 8.63 & 7.20 & 52.20 & 3.26 & 2.34 & 5.29 & 2.40 & 1.82 & 2.65 \\
\hline 1.8 & 20.26 & 9.35 & 7.46 & 63.46 & 3.37 & 2.48 & 5.88 & 2.26 & 1.77 & 2.23 \\
\hline 2.0 & 22.01 & 10.06 & 7.73 & 76.10 & 3.45 & 2.61 & 6.39 & 2.12 & 1.73 & 1.94 \\
\hline
\end{tabular}
$\theta_{A \xi}=\sum_{\xi \in \Omega} \rho_{\xi} \theta_{B \xi}=1$, Table 2 shows that as the market potential of product $\mathrm{A}$ increases, the wholesale and retail prices increase, while the demand is upward. Competitor demand despite price reduction has declined.

The price sensitivity parameter in comparison with the market potential has little effect on decision variables. According to Table 3, it can be assumed

Table 3. Effect of market potential on decision variables. 
Table 4. Effect of production cost on decision variables.

\begin{tabular}{|c|c|c|c|c|c|c|c|c|c|c|}
\hline \multirow[b]{2}{*}{$C_{A} / C_{B}$} & \multicolumn{4}{|c|}{ Retailer } & \multicolumn{3}{|c|}{ Manufacturer $A$} & \multicolumn{3}{|c|}{ Manufacturer $B$} \\
\hline & $S^{*}$ & $p_{A}^{*}$ & $p_{B}^{*}$ & $\prod_{r}$ & $\boldsymbol{w}_{A}^{*}$ & $q_{A}^{*}$ & $\prod_{A}$ & $\boldsymbol{w}_{B}^{*}$ & $q_{B}^{*}$ & $\prod_{B}$ \\
\hline 0.2 & 8.85 & 3.87 & 4.03 & 2.25 & 2.74 & 0.54 & 1.37 & 3.05 & 0.13 & 0.27 \\
\hline 0.4 & 8.87 & 3.93 & 4.04 & 2.09 & 2.86 & 0.50 & 1.23 & 3.07 & 0.18 & 0.37 \\
\hline 0.6 & 8.89 & 3.99 & 4.05 & 1.95 & 2.97 & 0.45 & 1.07 & 3.09 & 0.22 & 0.46 \\
\hline 0.8 & 8.91 & 4.03 & 4.06 & 1.84 & 3.06 & 0.39 & 0.88 & 3.11 & 0.28 & 0.59 \\
\hline 1.0 & 8.93 & 4.07 & 4.07 & 1.75 & 3.13 & 0.32 & 0.68 & 3.13 & 0.32 & 0.68 \\
\hline 1.2 & 8.95 & 4.10 & 4.08 & 1.68 & 3.19 & 0.26 & 0.64 & 3.15 & 0.44 & 0.95 \\
\hline 1.4 & 8.97 & 4.13 & 4.09 & 1.60 & 3.25 & 0.21 & 0.39 & 3.17 & 0.53 & 1.15 \\
\hline 1.6 & 8.99 & 4.17 & 4.10 & 1.51 & 3.34 & 0.13 & 0.23 & 3.19 & 0.62 & 1.36 \\
\hline 1.8 & 9.01 & 4.21 & 4.11 & 1.44 & 3.42 & 0.11 & 0.11 & 3.21 & 0.74 & 1.64 \\
\hline 2.0 & 9.03 & 4.26 & 4.11 & 1.37 & 3.51 & 0.03 & 0.03 & 3.22 & 0.86 & 1.91 \\
\hline
\end{tabular}

Table 5. Effect of price sensitivity on decision variables.

\begin{tabular}{|c|c|c|c|c|c|c|c|c|c|c|}
\hline \multirow[b]{2}{*}{$\frac{\sum_{\xi \in \Omega} \rho_{\xi} \theta_{A \xi}}{\sum_{\xi \in \Omega} \rho_{\xi} \theta_{B \xi}}$} & \multicolumn{4}{|c|}{ Retailer } & \multicolumn{3}{|c|}{ Manufacturer $A$} & \multicolumn{3}{|c|}{ Manufacturer $B$} \\
\hline & $S^{*}$ & $p_{A}^{*}$ & $p_{B}^{*}$ & $\prod_{r}$ & $w_{A}^{*}$ & $q_{A}^{*}$ & $\prod_{A}$ & $\boldsymbol{w}_{B}^{*}$ & $q_{B}^{*}$ & $\prod_{B}$ \\
\hline 0.2 & 9.01 & 3.94 & 3.90 & 2.33 & 2.81 & 0.56 & 1.01 & 2.87 & 0.96 & 1.80 \\
\hline 0.4 & 9.03 & 3.90 & 3.88 & 2.44 & 2.77 & 0.61 & 1.08 & 2.81 & 0.91 & 1.64 \\
\hline 0.6 & 9.05 & 3.88 & 3.86 & 2.57 & 2.71 & 0.68 & 1.16 & 2.76 & 0.87 & 1.53 \\
\hline 0.8 & 9.08 & 3.85 & 3.84 & 2.67 & 2.68 & 0.74 & 1.24 & 2.70 & 0.83 & 1.41 \\
\hline 1.0 & 9.10 & 3.82 & 3.82 & 2.81 & 2.63 & 0.79 & 1.29 & 2.63 & 0.79 & 1.29 \\
\hline 1.2 & 9.15 & 3.77 & 3.80 & 2.97 & 2.59 & 0.85 & 1.35 & 2.54 & 0.72 & 1.11 \\
\hline 1.4 & 9.18 & 3.75 & 3.77 & 3.09 & 2.54 & 0.91 & 1.40 & 2.49 & 0.64 & 0.95 \\
\hline 1.6 & 9.21 & 3.70 & 3.74 & 3.27 & 2.48 & 0.97 & 1.44 & 2.41 & 0.61 & 0.86 \\
\hline 1.8 & 9.26 & 3.68 & 3.72 & 3.40 & 2.43 & 1.03 & 1.48 & 2.35 & 0.57 & 0.77 \\
\hline 2.0 & 9.29 & 3.66 & 3.68 & 3.58 & 2.36 & 1.09 & 1.48 & 2.29 & 0.51 & 0.66 \\
\hline
\end{tabular}

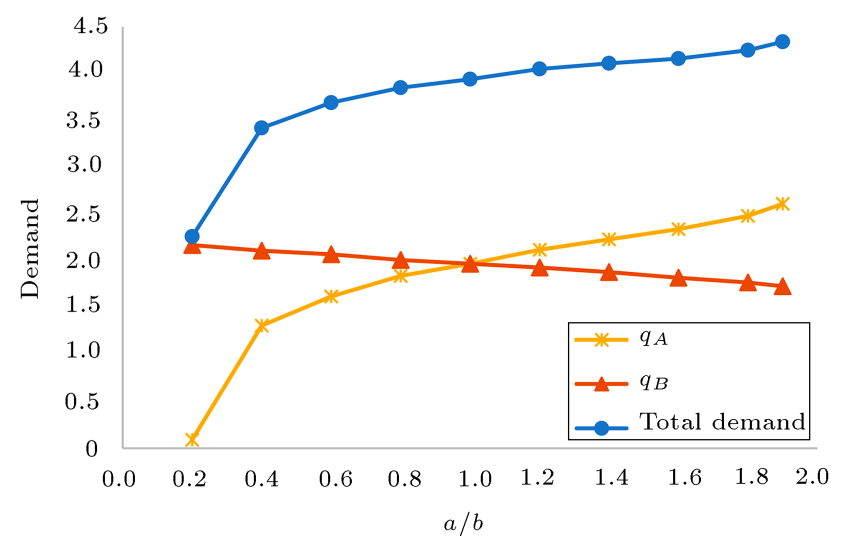

Figure 2. Effect of market potential ratio on demand.

that product $A$ is a luxury, in which the retailer profit and demand, despite price increment have been intensified.

As shown in Figure 2, despite the reduction in product $B$ demand, total demand increases and the retailer needs to increase the shelf space. It can be concluded that the market potential has a significant effect on demand and retailer and manufacturers profitability. Assuming:

$$
k=0.5, \quad b=5, \quad c_{B}=1, \quad \sum_{\xi \in \Omega} \rho_{\xi} \theta_{A \xi}=\sum_{\xi \in \Omega} \rho_{\xi} \theta_{B \xi}=1,
$$

Table 4 shows that wholesale and retail prices of both products increase by increasing the amount of production costs of product $A$. It can be seen that the growth trend of demand is related to the growth of retail prices. It can be concluded that the price sensitivity parameter is directly related to the production cost parameter. According to the values in Table 4 , products $A$ and $B$ are not in the luxury or giffen product group.

Despite a drop in demand for product $A$, the total demand for products is increasing. However, due to the increase in shelf space and related costs, the retailers profit margin drops. Manufacturers will produce a product at a lower wholesale price by reducing the amount of production costs through redesigning production processes and using new technologies. More 


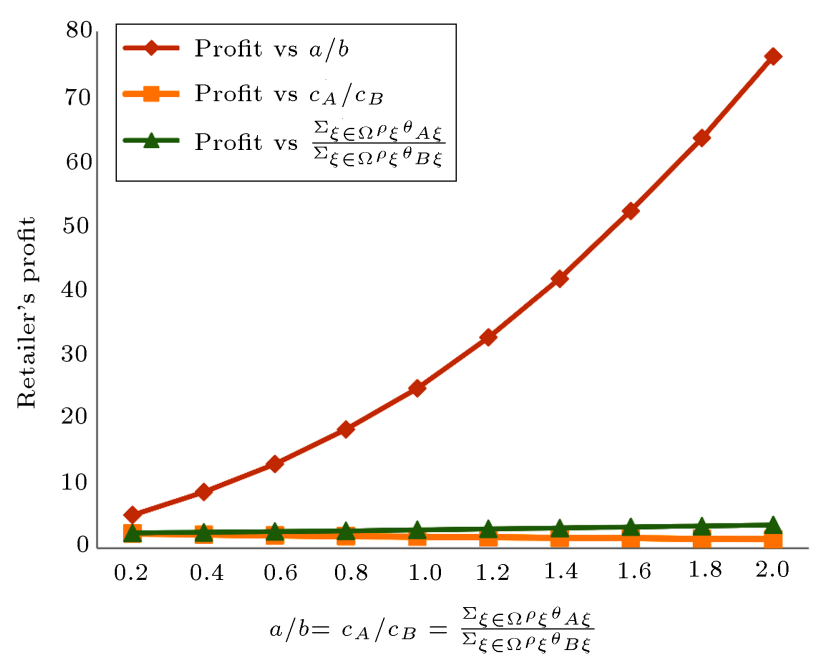

Figure 3. Effect of market potential, production cost, and price sensitivity on retailer profit.

interaction between manufacturers and retailers will provide more profit for all members of the supply chain.

Assuming $k=0.5, a=b=5, c_{A}=c_{B}=1$, $\sum_{\xi \in \Omega} \rho_{\xi} \theta_{B \xi}=0.5$, Table 5 shows that by increasing the average value of the price sensitivity parameter of product $A$, manufacturers and retailers reduce wholesale and retail prices. In this situation, a price-sensitive customer prefers the cheaper of the two products $A$ and $B$ and there is greater demand for a cheaper product. Indeed, in a situation where the price elasticity of demand is high, the amount of profits earned by the manufacturer and the retailer is associated with lower prices and cost savings. If the average price sensitivity parameter changes for different scenarios, the decision-making for supply chain members will be more conservative.

By increasing the average value of the sensitivity parameter, the certainty of the data is increased and the retailer places more shelf space on the product and earns more profit. The retailer with less shelf space and lower costs is struggling to make more profit, and manufacturers are trying to maintain their margins at higher prices.

Figure 3 shows that the retailer profit based on the ratio of the change in the parameters of the problem. Market potential has a significant positive effect on the profit margin earned by the retailer, and price sensitivity parameter and production cost have a negative impact on retailer profit. Manufacturers can increase the market potential of their products by offering new and unique products and increasing customer satisfaction.

\section{Conclusion}

This paper jointly optimizes pricing and shelf space allocation under uncertainty in a supply chain with two manufacturers and one retailer. Manufacturers have different conditions in terms of market potential, sensitivity parameters, and production cost. A robust optimization approach is used to model demand uncertainty and price sensitivity parameters. The most important results can be summarized as follows:

- By increasing the price sensitivity parameter, manufacturers and retailers reduce wholesale and retail prices. In this situation, there is greater demand for a cheaper product, compared to less sensitive cases;

- In uncertain circumstances, the decisions of the supply chain members will be more conservative. The retailer, with less shelf space and lower costs, is struggling to make more profit, and manufacturers are trying to maintain their margins at higher prices;

- Price sensitivity, market potential, and production costs can have a synergistic effect on optimal values. However, the intensity of the reciprocity of market potential optimal values is more than the other parameters. Therefore, this parameter is the most important tool for securing the profitability of supply chain members;

- When different scenarios have the same occurrence probability, the uncertainty of the data increases. In this situation, making decisions about pricing and shelf space allocation becomes more complicated for shelf space allocation becomes more complicated for supply chain members. Due to the fact that demand is not certain, the retailer makes a conservative decision in shelf space and considers similar pricing policies for both products. To minimize the losses, each manufacturer will try to make decisions that are similar to those of his competitors.

Further studies in this area can include the uncertainty surrounding other parameters of the problem such as market potential, production costs, and shelf space opportunity cost. Other approaches for considering uncertainty such as fuzzy logic can also be applied. Moreover, optimizing an extended multi-manufacturer multi-retailer supply chain can make future researches more attractive.

\section{References}

1. Anderson, E.E. and Amato, H.N., "A mathematical model for simultaneously determining the optimal brand-collection and display-area allocation", Operations Research, 22(1), pp. 13-21 (1974).

2. Corstjens, M. and Doyle, P. "A model for optimizing retail space allocation", Management Science, 27(7), pp. 822-833 (1981).

3. Corstjens, M. and Doyle, P. "A dynamic model for strategically allocating retail space", The Journal of 
the Operational Research Society, 34(10), pp. 943-951 (1983).

4. Bultez, A. and Naert, P. "Shelf allocation for retailers' profit", Marketing Science, 7(3), pp. 211-231 (1988).

5. Yang, M.H. "An efficient algorithm to allocate shelf space", European Journal of Operational Research, 131(1), pp. 107-118 (2001).

6. Hwang, H., Choi, B., and Lee, M.J. "A model for shelf space allocation and inventory control considering location and inventory level effects on demand", International Journal of Production Economics, 97, pp. 185-195 (2005).

7. Hansen, J.M., Raut, S., and Swami, S. "Retail shelf allocation: a comparative analysis of heuristic and metaheuristic approaches", Journal of Retailing, 86(1), pp. 94-105 (2010).

8. Castelli, M. and Vanneschi, L. "Genetic algorithm with variable neighborhood search for the optimal allocation of goods in shop shelves", Operations Research Letters, 42, pp. 355-360 (2014).

9. Zhou, W. and Piramuthu, S. "IoT and supply chain traceability", 1st International Conference on Future Network Systems and Security, FNSS, Paris, June, pp. 11-13 (2015).

10. Amit, R.K., Mehta, P., and Tripathi, R.P. "Optimal shelf-space stocking policy using stochastic dominance under supply-driven demand uncertainty", European Journal of Operational Research, 246(1), pp. 339-342 (2015).

11. Hübner, A. and Schaal, K. "An integrated assortment and shelf-space optimization model with demand substitution and space-elasticity effects", European Journal of Operational Research, 261(1), pp. 302-316 (2017).

12. Fatemi Ghomi, S.M.T. and Khalesi, S. "A hybrid approach for shelf space planning considering of stochastic demand and display facing area", 13th International Conference on Industrial Engineering (IIEC 2017) (2017).

13. Düsterhöft, T., Hübner, A., and Schaal, K. "A practical approach to the shelf-space allocation and replenishment problem with heterogeneously sized shelves", European Journal of Operational Research, 282(1), pp. 252-266 (2020).

14. Gajjar, H.K. and Adil, G.K. "A dynamic programming heuristic for retail shelf space allocation problem", Asia-Pacific Journal of Operational Research, 28(2), pp. 183-199 (2011).

15. Gilland, W.G. and Heese, H.S. "Sequence matters: shelf-space allocation under dynamic customer-driven substitution", Production and Operations Management, 22(4), pp. 875-887 (2013).
16. Martin-Herran, G. and Taboubi, S. "Shelf-space allocation and advertising decisions in the marketing channel: a differential game approach", International Game Theory Review, 7(3), pp. 313-330 (2005).

17. Hariga, M.A. and Al-Ahmari, A. "An integrated retail space allocation and lot sizing models under vendor managed inventory and consignment stock arrangements", Computers \& Industrial Engineering, 64(1), pp. $45-55$ (2013).

18. Tsao, Y.C., Lu, J.C., A.n, N., et al. "Retailer shelfspace management with trade allowance: A Stackelberg game between retailer and manufacturers", International Journal of Production Economics, 148, pp. 133-144 (2014).

19. Urban, T.L. "An inventory-theoretic approach to product assortment and shelf-space allocation", Journal of Retailing, 74(1), pp. 15-35 (1998).

20. Maiti, M.K. and Maiti, M. "Multi-item shelf-space allocation of breakable items via genetic algorithm", Journal of Applied Mathematics \& Computing, 20(1$2)$, pp. 327-343 (2006).

21. Hariga, M.A., Al-Ahmari, A., and Mohamed, A.A. "A joint optimisation model for inventory replenishment, product assortment, shelf space and display area allocation decisions", European Journal of Operational Research, 181(1), pp. 239-251 (2007).

22. Ramaseshan, B., Achuthan, N.R., and Collinson, R. "Decision support tool for retail shelf space optimization", International Journal of Information Technology \& Decision Making, 7(3), pp. 547-565 (2008).

23. Bai, R. and Kendall, G. "A model for fresh produce shelf-space allocation and inventory management with freshness-condition-dependent demand", Journal of Computing, 20(1), pp. 78-85 (2008).

24. Gao, J.J. and Yu, L.R. "Joint decision model of variants selection, shelf-space allocation and inventory control", Xitong Gongcheng Xuebao, 24(5), pp. 614620 (2009).

25. Baron, O., Berman, O., and Perry, D. "Shelf space management when demand depends on the inventory level", Production and Operations Management, 20(5), pp. 714-726 (2011).

26. Hwang, H., Choi, B., and Lee, G. "A genetic algorithm approach to an integrated 12 problem of shelf space design and item allocation", Computers \& Industrial Engineering, 56, pp. 809-820 (2009).

27. Ghazavi, E. and Lotfi, M.M. "Formulation of customers' shopping path in shelf space planning: A simulation-optimization approach", Expert Systems with Applications, 55, pp. 243-254 (2016).

28. Flamand, T., Ghoniem, A., Haouari, M., and Maddah, B. "Integrated assortment planning and storewide shelf space allocation: An optimization-based approach", Omega, 81, pp. 134-149 (2018). 
29. Schaal, K. and Hübner, A. "When does cross-space elasticity matter in shelf-space planning? A decision analytics approach", Omega, 80, pp. 135-152 (2018).

30. Shafiee-Gole, S., Nasiri, M., and Taleizadeh, A. "Pricing and production decisions in multi-product single machine manufacturing system with discrete delivery and rework", OPSEARCH (2016).

31. Sadjadi, S.J., Asadi, H., Sadeghian, R., et al. "Retailer Stackelberg game in a supply chain with pricing and service decisions and simple price discount contract", PLOS ONE, 13(4), e0195109 (2018). https://doi.org/10.1371/journal.pone.0195109

32. Reyes, P.M. and Frazier, G.V. "Goal programming model for grocery shelf space allocation", European Journal of Operational Research, 181(2), pp. 634-644 (2007).

33. Murray, C.C., Talukdar, D., and Gosavi, A. "Joint optimization of product price, display orientation and shelf-space allocation in retail category management, Journal of Retailing, 86(2), pp. 125-136 (2010).

34. Tan, M. and Wang, H. "Retailer's shelf-space and pricing decisions under revenue sharing contracts", International Conference on Management and Service Science (2011).

35. Kurtulus, M. and Toktay, L.B. "Category captainship vs. retailer category management under limited retail shelf space", Production and Operations Management Society, 20, pp. 47-56 (2011).

36. Leng, M., Parlar, M., and Zhang, D., Retail SpaceExchange Problem with Pricing and Space Allocation Decisions, Production and Operations Management Society, pp. 1-14 (2011).

37. Li, X. Nukal, S., and Moheb, S. "Game theory methodology for optimizing retailers' pricing and shelf-space allocation decisions on competing substitutable products", International Journal of Advanced Design and Manufacturing Technology, 68, pp. 375-389 (2013).

38. Eisend, M. "Shelf space elasticity: A meta-analysis", Journal of Retailing, 90(2), pp. 168-181 (2014).

39. Wang, S.Y., Sheen, G.J., and Yeh, Y. "Pricing and shelf space decisions with non-symmetric market demand", International Journal of Production Economics, 169, pp. 233-239 (2015).

40. Hübner, A. and Schaal, K. "A shelf-space optimization model when demand is stochastic and space-elastic", Omega, 68, pp. 139-154 (2017).

41. Moon, I., Park, K.S, Hao, J., and Kim, D. "Joint decisions on product line selection, purchasing, and pricing", European Journal of Operational Research, 262(1), pp. 207-216 (2017).
42. Li, H.L. "An efficient method for solving linear goal programming problems", Journal of Optimization Theory and Applications, 90, pp. 465-469 (1996).

43. Mirzapour, Al-e-hashem, S.M.J., Malekly, H., and Aryanezhad, M.B. "A multi-objective robust optimization model for multi-product multi-site aggregate production planning in a supply chain under uncertainty", International Journal of Production Economics, 134, pp. 28-42 (2011).

44. Mulvey, J.M., Vanderbei, R.J., and Zenios, S.A. "Robust optimization of large-scale systems", Operations Research, 43(2), pp. 264-281 (1995).

45. Feng, P. and Rakesh, N. "Robust supply chain design under uncertain demand in agile manufacturing", Computers \& Operations Research, 37(4), pp. 668-683 (2010).

46. Leung, S.C.H., Tsang, S.O.S., Ng., W.L., et al. "A robust optimization model for multi-site production planning problem in an uncertain environment, $E u$ ropean Journal of Operational Research, 181(1), pp. 224-238 (2007).

47. Wagner, H.M., Principles of Operations Research, Second Ed., Prentice Hall, New Jersey (1975).

48. Yu, C.S. and Li, H.L. "A robust optimization model for stochastic logistic problem", International Journal of Production Economics, 64(1-3), pp. 385-397 (2000).

49. Shubik, M. and Levitan, R., Market Structure and Behavior, Harvard University Press, Cambridge (1980).

\section{Appendix A}

The retailer robust objective function is as follows:

$$
\begin{aligned}
\prod_{n}= & (1-\lambda) \sum_{\xi \in \Omega} \rho_{\xi} Z_{\xi}+\lambda \\
& \left(\sum_{\xi \in \Omega} \sum_{\xi^{\prime} \in \Omega} \rho_{\xi} \rho_{\xi^{\prime}} Z_{\xi^{\prime}}\right)-2 \lambda \sum_{\xi \in \Omega} \rho_{\xi} u_{\xi} \\
& -\psi \sum_{\xi \in \Omega} \rho_{\xi} \varepsilon_{\xi} .
\end{aligned}
$$

To determine whether a function $f\left(x_{1}, x_{2}, \ldots, x_{n}\right)$ of $n$ variable is convex or concave on a set of $S \subset$ $R^{n}$, we assume that $f\left(x_{1}, x_{2}, \ldots, x_{n}\right)$ has continuous second-order partial derivatives. The Hessian of $f\left(x_{1}, x_{2}, \ldots, x_{n}\right)$ is a $n \times n$ matrix that its $i j$ th entry is $\frac{\partial^{2} f}{\partial_{x_{i}} \partial_{x_{j}}} \bullet$.

An $i$ th principal minor of a $n \times n$ matrix is the determinant of any $i \times i$ matrix obtained by deleting $n-i$ rows and the corresponding $n-i$ columns of the matrix. The $k$ th leading principal minor of a $n \times n$ matrix is the determinant of the $k \times k$ matrix obtained by deleting the last $n-k$ rows and columns of the matrix. The function $f\left(x_{1}, x_{2}, \ldots, x_{n}\right)$ is concave on $S$ 
if and only if for each $x \in S$ and $k=1,2, \ldots, n$, all nonzero principal minors have the same sign as $(-1)^{k}$. To obtain Gradient vector, we have:

$$
\begin{aligned}
& A=\frac{\partial \prod_{n}}{\partial p_{A}}=(1-\lambda) \\
& \sum_{\xi \in \Omega} \rho_{\xi}\left[a-2 p_{A}+\theta_{A \xi} p_{B}-2 p_{A} \theta_{A \xi}+w_{A}+w_{A} \theta_{A \xi}\right. \\
& \left.+p_{B} \theta_{B \xi}-w_{B} \theta_{B \xi}\right]+\lambda\left(\sum _ { \xi \in \Omega } \sum _ { \xi ^ { \prime } \in \Omega } \rho _ { \xi } \rho _ { \xi ^ { \prime } } \left[a-2 p_{A}\right.\right. \\
& +\theta_{A \xi^{\prime}} p_{B}-2 p_{A} \theta_{A \xi^{\prime}}+w_{A}+w_{A} \theta_{A \xi^{\prime}} \\
& \left.\left.+p_{B} \theta_{B \xi^{\prime}}-w_{B} \theta_{B \xi^{\prime}}\right]\right), \\
& B=\frac{\partial \prod_{n}}{\partial p_{B}}=(1-\lambda) \sum_{\xi \in \Omega} \rho_{\xi} \\
& {\left[p_{A} \theta_{A \xi}-w_{A} \theta_{A \xi}+b-2 p_{B}+\theta_{B \xi} p_{A}-2 p_{B} \theta_{B \xi}\right.} \\
& \left.+w_{B}+w_{B} \theta_{B \xi}\right]+\lambda\left(\sum _ { \xi \in \Omega \xi ^ { \prime } \in \Omega } \rho _ { \xi } \rho _ { \xi ^ { \prime } } \left[p_{A} \theta_{A \xi^{\prime}}-w_{A} \theta_{A \xi^{\prime}}\right.\right. \\
& \left.\left.+b-2 p_{B}+\theta_{B \xi^{\prime}} p_{A}-2 p_{B} \theta_{B \xi^{\prime}}+w_{B}+w_{B} \theta_{B \xi^{\prime}}\right]\right), \\
& \frac{\partial \prod_{n}}{\partial u_{\xi}}=-2 \lambda \sum_{\xi \in \Omega} \rho_{\xi}, \\
& \frac{\partial \prod_{n}}{\partial \varepsilon_{\xi}}=-H \sum_{\xi \in \Omega} \rho_{\xi} .
\end{aligned}
$$

The Gradient vector is as follows:

$$
\nabla \prod_{n}=\left[\begin{array}{c}
A \\
B \\
-2 \lambda \rho_{1} \\
-2 \lambda \rho_{2} \\
-2 \lambda \rho_{3} \\
\cdot \\
\cdot \\
-2 \lambda \rho_{t} \\
H \rho_{1} \\
H \rho_{2} \\
H \rho_{3} \\
\cdot \\
\cdot \\
\cdot \\
H \rho_{\xi}
\end{array}\right] .
$$

To obtain the Hessian matrix, we have:

$$
\begin{aligned}
& C=\frac{\partial^{2} \prod_{n}}{\partial p^{2}{ }_{A}}=(1-\lambda) \sum_{\xi \in \Omega} \rho_{\xi}\left[-2-2 \theta_{A \xi}\right] \\
& +\lambda\left(\sum_{\xi \in \Omega} \sum_{\xi^{\prime} \in \Omega} \rho_{\xi} \rho_{\xi^{\prime}}\left[-2-2 \theta_{A \xi^{\prime}}\right]\right), \\
& D=\frac{\partial^{2} \prod_{n}}{\partial p_{A} p_{B}}=(1-\lambda) \sum_{\xi \in \Omega} \rho_{\xi}\left[\theta_{A \xi}+\theta_{B \xi}\right] \\
& +\lambda\left(\sum_{\xi \in \Omega} \sum_{\xi^{\prime} \in \Omega} \rho_{\xi} \rho_{\xi^{\prime}}\left[\theta_{A \xi^{\prime}}+\theta_{B \xi^{\prime}}\right]\right), \\
& \frac{\partial^{2} \prod_{n}}{\partial p_{A} u_{\xi}}=0, \\
& \frac{\partial^{2} \prod_{n}}{\partial p_{B} \xi_{\xi}}=0, \\
& \frac{\partial^{2} \prod_{n}}{\partial u_{\xi}^{2}}=0, \\
& \frac{\partial^{2} \prod_{n}}{\partial \varepsilon_{\xi}^{2}}=0, \\
& \frac{\partial^{2} \prod_{n}}{\partial u_{\xi} p_{A}}=0, \\
& \frac{\partial^{2} \prod_{n}}{\partial u_{\xi} p_{B}}=0, \\
& \frac{\partial^{2} \prod_{n}}{\partial \xi_{\xi} p_{A}}=0, \\
& \frac{\partial^{2} \prod_{n}}{\partial \xi_{\xi} p_{B}}=0 .
\end{aligned}
$$

The Hessian matrix is as follows:

Hessian matrix $=$

$$
\left[\begin{array}{ccccc}
C & D & \cdots & 0 & 0 \\
F & E & & 0 & 0 \\
0 & 0 & \ddots & 0 & 0 \\
\vdots & \vdots & & \vdots & \vdots \\
0 & 0 & \cdots & 0 & 0
\end{array}\right]_{(2 \xi+2) \times(2 \xi+2)}
$$

If $C<0$ and $\left|\begin{array}{cc}C & D \\ F & E\end{array}\right|>0$, it can be concluded that the objective function is concave and has a globally optimized solution.

The proof of objective function concavity can be presented using the following steps: 
1. We show the first principal minors $(C)$ is nonpositive, i.e., $(-C>0)$ or $(C<0)$ :

$$
\begin{aligned}
-C= & (1-\lambda) \sum_{\xi \in \Omega} \rho_{\xi}\left[2+2 \theta_{A \xi}\right] \\
& +\lambda\left(\sum_{\xi \in \Omega} \sum_{\xi^{\prime} \in \Omega} \rho_{\xi} \rho_{\xi^{\prime}}\left[2+2 \theta_{A \xi^{\prime}}\right]\right)>0 .
\end{aligned}
$$

We know that:

$$
\sum_{\xi \in \Omega} \rho_{\xi}=1, \quad \sum_{\xi \in \Omega} \rho_{\xi} \theta_{A \xi} \leq 1, \quad \sum_{\xi^{\prime} \in \Omega} \rho_{\xi^{\prime}} \theta_{A \xi^{\prime}} \leq 1 .
$$

Thus:

$$
\begin{aligned}
& -C / 2=(1-\lambda) \sum_{\xi \in \Omega} \rho_{\xi}\left[1+\theta_{A \xi}\right] \\
& +\lambda\left(\sum_{\xi \in \Omega} \sum_{\xi^{\prime} \in \Omega} \rho_{\xi} \rho_{\xi^{\prime}}\left[1+\theta_{A \xi^{\prime}}\right]\right)>0, \\
& =\sum_{\xi \in \Omega} \rho_{\xi}\left[1+\theta_{A \xi}\right]-\lambda \sum_{\xi \in \Omega} \rho_{\xi} \\
& \left(1+\theta_{A \xi}-\sum_{\xi^{\prime} \in \Omega} \rho_{\xi^{\prime}}\left[1+\theta_{A \xi^{\prime}}\right]\right)>0, \\
& =\sum_{\xi \in \Omega} \rho_{\xi}+\sum_{\xi \in \Omega} \theta_{A \xi} \rho_{\xi}-\lambda \\
& \left(\sum_{\xi \in \Omega} \rho_{\xi}+\sum_{\xi \in \Omega} \theta_{A \xi} \rho_{\xi}+\sum_{\xi \in \Omega} \rho_{\xi} \sum_{\xi^{\prime} \in \Omega} \rho_{\xi^{\prime}}\left[1+\theta_{A \xi^{\prime}}\right]\right) \\
& >0=1+\sum_{\xi \in \Omega} \theta_{A \xi} \rho_{\xi}-\lambda \\
& \left(1+\sum_{\xi \in \Omega} \theta_{A \xi} \rho_{\xi}+\sum_{\xi^{\prime} \in \Omega} \rho_{\xi^{\prime}}\left[1+\theta_{A \xi^{\prime}}\right]\right)>0, \\
& =\lambda\left(1+\sum_{\xi \in \Omega} \theta_{A \xi} \rho_{\xi}+\sum_{\xi^{\prime} \in \Omega} \rho_{\xi^{\prime}}\left[1+\theta_{A \xi^{\prime}}\right]\right) \\
& <1+\sum_{\xi \in \Omega} \theta_{A \xi} \rho_{\xi}, \quad \text { if : } \\
& \sum_{\xi \in \Omega} \theta_{A \xi} \rho_{\xi}=0 \rightarrow \sum_{\xi^{\prime} \in \Omega} \rho_{\xi^{\prime}} \theta_{A \xi^{\prime}}=0 .
\end{aligned}
$$

$$
\begin{aligned}
& B=D=2(1-\lambda)+2 \lambda=2, \\
& C=-4(1-\lambda)-4 \lambda=-4 .
\end{aligned}
$$

Then:

$$
C E-D F=12>0 .
$$

For minimum value we will have $\theta_{A \xi}=0, \theta_{B \xi}=0$, $\rho_{\xi}=1$. Thus:

$$
\begin{aligned}
& A=-2(1-\lambda)-2 \lambda=-2, \\
& B=D=0, \\
& C=-2(1-\lambda)-2 \lambda=-2 .
\end{aligned}
$$

Then:

$$
C E-D F=4>0 .
$$

Therefore, $C E-D F$ will be positive under all scenarios and for all values between maximum and minimum. Based on $(i)$, (ii), and as $|H|=0$, we can finally conclude that the objective function is concave and the software optimized solution is global.

\section{Appendix B}

The auxiliary expressions used in Eq. (28) are as follows:

$$
\begin{aligned}
E_{1}= & -8260 \sum_{\xi \in \Omega} \rho_{\xi} \theta_{A \xi}-11340 \sum_{\xi \in \Omega} \rho_{\xi} \theta_{B \xi} \\
& -19600 \sum_{\xi \in \Omega} \rho_{\xi} \theta_{A \xi} \theta_{B \xi}-4779,
\end{aligned}
$$

$1+2 \rightarrow \lambda<\frac{1}{2}$. We then find that $-C>0$ or $C<0$ holds if $0<\lambda<\frac{1}{2} \bullet$ 


$$
\begin{aligned}
E_{2}= & 7296 \sum_{\xi \in \Omega} \rho_{\xi} \theta_{A \xi}+21708 \sum_{\xi \in \Omega} \rho_{\xi} \theta_{B \xi} \\
& +17152 \sum_{\xi \in \Omega} \rho_{\xi} \theta_{A \xi} \theta_{B \xi}+9234 \\
E_{3}= & 15390 \sum_{\xi \in \Omega} \rho_{\xi} \theta_{B \xi}^{2}-1980 \sum_{\xi \in \Omega} \rho_{\xi} \theta_{A \xi} \theta_{B \xi} \\
& +25460 \sum_{\xi \in \Omega} \rho_{\xi} \theta_{A \xi} \theta_{B \xi}^{2}-7808 \sum_{\xi \in \Omega} \rho_{\xi} \theta_{B \xi} \theta_{A \xi}^{2} \\
& -4277 \sum_{\xi \in \Omega} \rho_{\xi} \theta_{A \xi} \theta_{B \xi}+15390 \sum_{\xi \in \Omega} \rho_{\xi} \theta_{B \xi} \\
& -5959 \sum_{\xi \in \Omega} \rho_{\xi} \theta_{A \xi},
\end{aligned}
$$$$
E_{4}=54376 \sum_{\xi \in \Omega} \rho_{\xi} \theta_{B \xi}-(1.14)^{5} \sum_{\xi \in \Omega} \rho_{\xi} \theta_{A \xi}
$$$$
-5496 \sum_{\xi \in \Omega} \rho_{\xi} \theta_{A \xi} \theta_{B \xi}-(1.18)^{5},
$$$$
E_{5}=(1.11)^{5} \sum_{\xi \in \Omega} \rho_{\xi} \theta_{B \xi}+10015 \sum_{\xi \in \Omega} \rho_{\xi} \theta_{A \xi}
$$$$
+29212 \sum_{\xi \in \Omega} \rho_{\xi} \theta_{A \xi} \theta_{B \xi}-49685,
$$$$
E_{6}=71306 \sum_{\xi \in \Omega} \rho_{\xi} \theta_{B \xi}+53687 \sum_{\xi \in \Omega} \rho_{\xi} \theta_{A \xi}
$$$$
+14204 \sum_{\xi \in \Omega} \rho_{\xi} \theta_{A \xi} \theta_{B \xi}+90422,
$$

$$
\begin{aligned}
E_{7}= & 0.077-0.02 \sum_{\xi \in \Omega} \rho_{\xi} \theta_{A \xi}, \\
E_{8}= & 0.162-0.128 \sum_{\xi \in \Omega} \rho_{\xi} \theta_{A \xi}, \\
E_{9}= & 37104 \sum_{\xi \in \Omega} \rho_{\xi} \theta_{A \xi} \theta_{B \xi}+15772 \sum_{\xi \in \Omega} \rho_{\xi} \theta_{A \xi} \\
& +32636 \sum_{\xi \in \Omega} \rho_{\xi} \theta_{B \xi}+13925,
\end{aligned}
$$$$
\begin{aligned}
E_{10}= & -a\left(0.139+0.458 \sum_{\xi \in \Omega} \rho_{\xi} \theta_{A \xi}\right) \\
& -b\left(0.919-0.109 \sum_{\xi \in \Omega} \rho_{\xi} \theta_{A \xi}\right) \\
& +S\left(0.448-0.053 \sum_{\xi \in \Omega} \rho_{\xi} \theta_{A \xi}\right) \\
& -c_{A}\left(0.081+0.14 \sum_{\xi \in \Omega} \rho_{\xi} \theta_{A \xi}\right) \\
& -0.101 \sum_{\xi \in \Omega} \rho_{\xi} \theta_{A \xi}+0.095 \sum_{\xi \in \Omega} \rho_{\xi} \theta_{A \xi} \theta_{B \xi} .
\end{aligned}
$$

The auxiliary expressions used in Eq. (29) are as follows:

$$
\begin{gathered}
F_{1}=157772 \sum_{\xi \in \Omega} \rho_{\xi} \theta_{A \xi}-32636 \sum_{\xi \in \Omega} \rho_{\xi} \theta_{B \xi} \\
-337104 \sum_{\xi \in \Omega} \rho_{\xi} \theta_{A \xi} \theta_{B \xi},
\end{gathered}
$$

$$
\begin{gathered}
F_{2}=-11340 \sum_{\xi \in \Omega} \rho_{\xi} \theta_{B \xi}-8260 \sum_{\xi \in \Omega} \rho_{\xi} \theta_{A \xi} \\
-19600 \sum_{\xi \in \Omega} \rho_{\xi} \theta_{A \xi} \theta_{B \xi}-4779
\end{gathered}
$$

$$
\begin{gathered}
F_{3}=21708 \sum_{\xi \in \Omega} \rho_{\xi} \theta_{B \xi}+7296 \sum_{\xi \in \Omega} \rho_{\xi} \theta_{A \xi} \\
-17152 \sum_{\xi \in \Omega} \rho_{\xi} \theta_{A \xi} \theta_{B \xi}+9234
\end{gathered}
$$

$$
\begin{aligned}
F_{4}= & -15390 \sum_{\xi \in \Omega} \rho_{\xi} \theta_{B \xi}-5959 \sum_{\xi \in \Omega} \rho_{\xi} \theta_{A \xi} \\
& -6277 \sum_{\xi \in \Omega} \rho_{\xi} \theta_{A \xi} \theta_{B \xi}+15390 \sum_{\xi \in \Omega} \rho_{\xi} \theta_{B \xi}^{2}
\end{aligned}
$$$$
+25460 \sum_{\xi \in \Omega} \rho_{\xi} \theta_{B \xi}^{2} \theta_{A \xi}-7808 \sum_{\xi \in \Omega} \rho_{\xi} \theta_{A \xi}^{2} \theta_{B \xi},
$$$$
F_{5}=54736 \sum_{\xi \in \Omega} \rho_{\xi} \theta_{B \xi}-(1.14)^{5} \sum_{\xi \in \Omega} \rho_{\xi} \theta_{A \xi}
$$

$$
-5496 \sum_{\xi \in \Omega} \rho_{\xi} \theta_{A \xi} \theta_{B \xi}-(1.18)^{5},
$$




$$
\begin{gathered}
F_{6}=-(1.15)^{5} \sum_{\xi \in \Omega} \rho_{\xi} \theta_{B \xi}+10015 \sum_{\xi \in \Omega} \rho_{\xi} \theta_{A \xi} \\
+29212 \sum_{\xi \in \Omega} \rho_{\xi} \theta_{A \xi} \theta_{B \xi}-49685 \\
F_{7}=71306 \sum_{\xi \in \Omega} \rho_{\xi} \theta_{B \xi}+53687 \sum_{\xi \in \Omega} \rho_{\xi} \theta_{A \xi} \\
\quad+14204 \sum_{\xi \in \Omega} \rho_{\xi} \theta_{A \xi} \theta_{B \xi}+90422,
\end{gathered}
$$

The auxiliary expressions used in Eq. (31) are as follows:

$$
\begin{aligned}
& G_{1}=(1.16)^{14} \sum_{\xi \in \Omega} \rho_{\xi} \theta_{A \xi}^{3}+(2.05)^{14} \sum_{\xi \in \Omega} \rho_{\xi} \theta_{B \xi}^{3} \\
& +(2.1)^{14} \sum_{\xi \in \Omega} \rho_{\xi} \theta_{A \xi}^{2}+(5.55)^{14} \sum_{\xi \in \Omega} \rho_{\xi} \theta_{B \xi}^{2} \\
& +(2.16)^{14} \sum_{\xi \in \Omega} \rho_{\xi} \theta_{A \xi}+(3.18)^{14} \sum_{\xi \in \Omega} \rho_{\xi} \theta_{B \xi} \\
& +(7.7)^{13}-(5.21)^{12} \sum_{\xi \in \Omega} \rho_{\xi} \theta_{A \xi}^{3} \theta_{B \xi}^{2} \\
& +(3.37)^{13} \sum_{\xi \in \Omega} \rho_{\xi} \theta_{A \xi}^{2} \theta_{B \xi}^{3}+(2.7)^{14} \sum_{\xi \in \Omega} \rho_{\xi} \theta_{A \xi}^{3} \theta_{B \xi} \\
& +(3.05)^{14} \sum_{\xi \in \Omega} \rho_{\xi} \theta_{A \xi}^{2} \theta_{B \xi}^{2}+(3.75)^{14} \sum_{\xi \in \Omega} \rho_{\xi} \theta_{A \xi} \theta_{B \xi}^{3} \\
& +(6.2)^{14} \sum_{\xi \in \Omega} \rho_{\xi} \theta_{A \xi}^{2} \theta_{B \xi}+(1.13)^{15} \sum_{\xi \in \Omega} \rho_{\xi} \theta_{A \xi} \theta_{B \xi}^{2} \\
& +(9.25)^{14} \sum_{\xi \in \Omega} \rho_{\xi} \theta_{A \xi} \theta_{B \xi}, \\
& G_{2}=(6.76)^{12} \sum_{\xi \in \Omega} \rho_{\xi} \theta_{A \xi}^{3}-(4.23)^{11} \sum_{\xi \in \Omega} \rho_{\xi} \theta_{B \xi}^{3} \\
& -(1.39)^{14} \sum_{\xi \in \Omega} \rho_{\xi} \theta_{A \xi}^{2}-(3.4)^{14} \sum_{\xi \in \Omega} \rho_{\xi} \theta_{B \xi}^{2} \\
& -(1.75)^{14} \sum_{\xi \in \Omega} \rho_{\xi} \theta_{A \xi}-(3.4)^{14} \sum_{\xi \in \Omega} \rho_{\xi} \theta_{B \xi} \\
& -(8.33)^{13}-(7.73)^{13} \sum_{\xi \in \Omega} \rho_{\xi} \theta_{A \xi}^{3} \theta_{B \xi}^{2} \\
& +(1.1)^{14} \sum_{\xi \in \Omega} \rho_{\xi} \theta_{A \xi}^{2} \theta_{B \xi}^{3}-(4.86)^{14} \sum_{\xi \in \Omega} \rho_{\xi} \theta_{A \xi}^{2} \theta_{B \xi} \\
& -(3.52)^{14} \sum_{\xi \in \Omega} \rho_{\xi} \theta_{A \xi} \theta_{B \xi}^{2}-(6)^{14} \sum_{\xi \in \Omega} \rho_{\xi} \theta_{A \xi} \theta_{B \xi},
\end{aligned}
$$

$$
\begin{aligned}
& G_{3}=(6.7)^{14} \sum_{\xi \in \Omega} \rho_{\xi} \theta_{A \xi}^{3}-(8.2)^{14} \sum_{\xi \in \Omega} \rho_{\xi} \theta_{B \xi}^{3} \\
& +(3.97)^{15} \sum_{\xi \in \Omega} \rho_{\xi} \theta_{A \xi}^{2}+(1.12)^{15} \sum_{\xi \in \Omega} \rho_{\xi} \theta_{B \xi}^{2} \\
& +(5.4)^{15} \sum_{\xi \in \Omega} \rho_{\xi} \theta_{A \xi}+(5.37)^{15} \sum_{\xi \in \Omega} \rho_{\xi} \theta_{B \xi} \\
& +(2.35)^{15}+(3.1)^{14} \sum_{\xi \in \Omega} \rho_{\xi} \theta_{A \xi}^{3} \theta_{B \xi}^{2} \\
& -(5.64)^{13} \sum_{\xi \in \Omega} \rho_{\xi} \theta_{A \xi}^{2} \theta_{B \xi}^{3}+(1.99)^{15} \sum_{\xi \in \Omega} \rho_{\xi} \theta_{A \xi}^{3} \theta_{B \xi} \\
& +(2.25)^{15} \sum_{\xi \in \Omega} \rho \theta_{A \xi}^{2} \theta_{B \xi}^{2}+(1.45)^{14} \sum_{\xi \in \Omega} \rho_{\xi} \theta_{A \xi} \theta_{B \xi}^{3} \\
& +(8.04)^{15} \sum_{\xi \in \Omega} \rho_{\xi} \theta_{A \xi}^{2} \theta_{B \xi}+(4.56)^{15} \sum_{\xi \in \Omega} \rho_{\xi} \theta_{A \xi} \theta_{B \xi}^{2} \\
& +(1.86)^{16} \sum_{\xi \in \Omega} \rho_{\xi} \theta_{A \xi} \theta_{B \xi}, \\
& G_{4}=\sum_{\xi \in \Omega} \rho_{\xi} \theta_{A \xi}^{3}+(2.85)^{15} \sum_{\xi \in \Omega} \rho_{\xi} \theta_{B \xi}^{3} \\
& +(9.2)^{14} \sum_{\xi \in \Omega} \rho_{\xi} \theta_{A \xi}^{2}+(1.14)^{16} \sum_{\xi \in \Omega} \rho_{\xi} \theta_{B \xi}^{2} \\
& +(2.47)^{15} \sum_{\xi \in \Omega} \rho_{\xi} \theta_{A \xi}+(9.42)^{15} \sum_{\xi \in \Omega} \rho_{\xi} \theta_{B \xi} \\
& -(1.1)^{14} \sum_{\xi \in \Omega} \rho_{\xi} \theta_{A \xi}^{3} \theta_{B \xi}^{2}+(1.02)^{14} \sum_{\xi \in \Omega} \rho_{\xi} \theta_{A \xi}^{2} \theta_{B \xi}^{3} \\
& -(1.75)^{15} \sum_{\xi \in \Omega} \rho_{\xi} \theta_{A \xi}^{3} \theta_{B \xi}+(7.49)^{14} \sum_{\xi \in \Omega} \rho_{\xi} \theta_{A \xi}^{2} \theta_{B \xi}^{2} \\
& +(7.45)^{14} \sum_{\xi \in \Omega} \rho_{\xi} \theta_{A \xi} \theta_{B \xi}^{3}+(2.41)^{15} \sum_{\xi \in \Omega} \rho_{\xi} \theta_{A \xi}^{2} \theta_{B \xi} \\
& +(8.35)^{15} \sum_{\xi \in \Omega} \rho_{\xi} \theta_{A \xi} \theta_{B \xi}^{2},
\end{aligned}
$$




$$
\begin{aligned}
& G_{5}=-(9.4)^{13} \sum_{\xi \in \Omega} \rho_{\xi} \theta_{A \xi}^{3}-(9.7)^{14} \sum_{\xi \in \Omega} \rho_{\xi} \theta_{B \xi}^{3} \\
& -(2.5)^{14} \sum_{\xi \in \Omega} \rho_{\xi} \theta_{A \xi}^{2}-(9.53)^{14} \sum_{\xi \in \Omega} \rho_{\xi} \theta_{B \xi}^{2} \\
& -(9.32)^{15} \sum_{\xi \in \Omega} \rho_{\xi} \theta_{A \xi}-(2.7)^{14} \sum_{\xi \in \Omega} \rho_{\xi} \theta_{B \xi} \\
& -(1.28)^{13} \sum_{\xi \in \Omega} \rho_{\xi} \theta_{A \xi}^{3} \theta_{B \xi}^{2}-(4.07)^{14} \sum_{\xi \in \Omega} \rho_{\xi} \theta_{A \xi}^{3} \theta_{B \xi}^{2} \\
& -(3.09)^{14} \sum_{\xi \in \Omega} \rho_{\xi} \theta_{A \xi}^{2} \theta_{B \xi}^{3}-(4.08)^{14} \sum_{\xi \in \Omega} \rho_{\xi} \theta_{A \xi}^{3} \theta_{B \xi} \\
& -(7.47)^{14} \sum_{\xi \in \Omega} \rho_{\xi} \theta_{A \xi}^{2} \theta_{B \xi}^{2}-(4.7)^{14} \sum_{\xi \in \Omega} \rho_{\xi} \theta_{A \xi} \theta_{B \xi}^{3} \\
& -(6.5)^{14} \sum_{\xi \in \Omega} \rho_{\xi} \theta_{A \xi} \theta_{B \xi}^{3}-(1.01)^{15} \sum_{\xi \in \Omega} \rho_{\xi} \theta_{A \xi}^{2} \theta_{B \xi} \\
& +(8.9)^{14} \sum_{\xi \in \Omega} \rho_{\xi} \theta_{A \xi}^{2} \theta_{B \xi}-(7.7)^{14} \sum_{\xi \in \Omega} \rho_{\xi} \theta_{A \xi} \theta_{B \xi}^{2} \\
& -(2.91)^{14} \sum_{\xi \in \Omega} \rho_{\xi} \theta_{A \xi} \theta_{B \xi}^{2}-(1.22)^{12} \sum_{\xi \in \Omega} \rho_{\xi} \theta_{A \xi} \theta_{B \xi} \\
& -(1.11)^{14} \sum_{\xi \in \Omega} \rho_{\xi} \theta_{A \xi}^{2} \theta_{B \xi}^{2}+(2.85)^{14} \sum_{\xi \in \Omega} \rho_{\xi} \theta_{A \xi}^{2} \theta_{B \xi}^{3} \\
& -(7.5)^{13} \sum_{\xi \in \Omega} \rho_{\xi} \theta_{A \xi}^{4} \theta_{B \xi}^{2}+(1.18)^{14} \sum_{\xi \in \Omega} \rho_{\xi} \theta_{A \xi}^{3} \theta_{B \xi}^{3} \\
& -(2.28)^{13} \sum_{\xi \in \Omega} \rho_{\xi} \theta_{A \xi}^{2} \theta_{B \xi}^{4}-(4.63)^{13} \sum_{\xi \in \Omega} \rho_{\xi} \theta_{A \xi}^{3} \theta_{B \xi}^{3} \\
& +(3.52)^{13} \sum_{\xi \in \Omega} \rho_{\xi} \theta_{A \xi}^{4} \theta_{B \xi}^{2}-(2.23)^{13} \sum_{\xi \in \Omega} \rho_{\xi} \theta_{A \xi}^{4} \\
& -(2.9)^{14} \sum_{\xi \in \Omega} \rho_{\xi} \theta_{B \xi}^{4}-(1.67)^{13} \sum_{\xi \in \Omega} \rho_{\xi} \theta_{A \xi}^{2} \theta_{B \xi}^{4} \\
& -(8.44)^{13} \sum_{\xi \in \Omega} \rho_{\xi} \theta_{A \xi}^{4} \theta_{B \xi}+(7.24)^{12} \sum_{\xi \in \Omega} \rho_{\xi} \theta_{A \xi}^{4} \theta_{B \xi} \\
& +(6.98)^{13} \sum_{\xi \in \Omega} \rho_{\xi} \theta_{A \xi}^{3} \theta_{B \xi}-(2.56)^{14} \sum_{\xi \in \Omega} \rho_{\xi} \theta_{A \xi} \theta_{B \xi}^{4} \\
& -(2.41)^{14} \sum_{\xi \in \Omega} \rho_{\xi} \theta_{A \xi} \theta_{B \xi}^{4}-(5.24)^{14} \sum_{\xi \in \Omega} \rho_{\xi} \theta_{A \xi} \theta_{B \xi},
\end{aligned}
$$

$$
\begin{aligned}
G_{6}= & (2.48)^{14} \sum_{\xi \in \Omega} \rho_{\xi} \theta_{A \xi}^{2}+(1.06)^{15} \sum_{\xi \in \Omega} \rho_{\xi} \theta_{B \xi}^{4} \\
& +(4.39)^{14} \sum_{\xi \in \Omega} \rho_{\xi} \theta_{A \xi}+(9.09)^{14} \sum_{\xi \in \Omega} \rho_{\xi} \theta_{B \xi} \\
& +(1.37)^{15} \sum_{\xi \in \Omega} \rho_{\xi} \theta_{A \xi}^{2} \theta_{B \xi}^{2}+(1.17)^{15} \sum_{\xi \in \Omega} \rho_{\xi} \theta_{A \xi}^{2} \theta_{B \xi} \\
& +(2.42)^{15} \sum_{\xi \in \Omega} \rho_{\xi} \theta_{A \xi} \theta_{B \xi}^{2}+(2.06)^{15} \sum_{\xi \in \Omega} \rho_{\xi} \theta_{A \xi} \theta_{B \xi} \\
& +(1.93)^{14}, \quad \text { (B.23) }
\end{aligned}
$$$$
G_{7}=(7.87)^{13} \sum_{\xi \in \Omega} \rho_{\xi} \theta_{A \xi}^{3}+(5.33)^{14} \sum_{\xi \in \Omega} \rho_{\xi} \theta_{B \xi}^{3}
$$$$
+(1.02)^{15} \sum_{\xi \in \Omega} \rho_{\xi} \theta_{A \xi}^{2}+(3.21)^{15} \sum_{\xi \in \Omega} \rho_{\xi} \theta_{B \xi}^{2}
$$$$
+(1.77)^{15} \sum_{\xi \in \Omega} \rho_{\xi} \theta_{A \xi}+(3.63)^{15} \sum_{\xi \in \Omega} \rho_{\xi} \theta_{B \xi}
$$$$
+(1.07)^{15}+(6.05)^{13} \sum_{\xi \in \Omega} \rho_{\xi} \theta_{A \xi}^{3} \theta_{B \xi}^{2}
$$$$
-(5.85)^{13} \sum_{\xi \in \Omega} \rho_{\xi} \theta_{A \xi}^{2} \theta_{B \xi}^{3}+(2.66)^{14} \sum_{\xi \in \Omega} \rho_{\xi} \theta_{A \xi}^{3} \theta_{B \xi}
$$$$
+(6.54)^{14} \sum_{\xi \in \Omega} \rho_{\xi} \theta_{A \xi}^{2} \theta_{B \xi}^{2}-(1.8)^{12} \sum_{\xi \in \Omega} \rho_{\xi} \theta_{A \xi} \theta_{B \xi}^{3}
$$$$
+(2.22)^{15} \sum_{\xi \in \Omega} \rho_{\xi} \theta_{A \xi}^{2} \theta_{B \xi}+(2.48)^{15} \sum_{\xi \in \Omega} \rho_{\xi} \theta_{A \xi} \theta_{B \xi}^{2}
$$$$
+(4.79)^{15} \sum_{\xi \in \Omega} \rho_{\xi} \theta_{A \xi} \theta_{B \xi} .
$$

\section{Biographies}

Mohsen S. Sajadieh received his $\mathrm{PhD}$ degree in Industrial Engineering, in 2009, from Sharif University of Technology, Tehran, Iran. He is now an Assistant Professor at the Amirkabir University of Technology. $\mathrm{He}$ is the author and co-author of more than 40 technical papers and the author of 5 books on the topics in the area of industrial engineering. His research area is focused on the supply chain. He is also interested in retailing management, stochastic modeling, and mathematical optimization.

Mohsen Danaei holds MSc in Industrial Engineering from the Amirkabir University of Technology and is an Assistant Lecturer for the marketing and MBA section of its management department. His main 
area of interest is the study of optimization and SCM problems. He dedicated his master theses to optimize SCM problems in uncertain circumstances. $\mathrm{He}$ is working on his thesis in the area of combining marketing elements and optimization approaches that take Artificial Intelligence (AI) tools into account. $\mathrm{He}$ is presently a member of Iran Elites National Foundation and a $\mathrm{PhD}$ candidate in the Amirkabir University of Technology, Department of Industrial Engineering. 